\title{
Vibration-induced Kondo tunneling through metal-organic complexes with even electron occupation number
}

\author{
K. Kikoin, ${ }^{1}$ M.N. Kiselev, ${ }^{2}$ and M.R. Wegewijs ${ }^{3}$ \\ ${ }^{1}$ Physics Department, Ben-Gurion University of the Negev, Beer-Sheva 84105, Israel \\ ${ }^{2}$ Institute für Theoretische Physik, Universität Würzburg, 97074 Würzburg, Germany \\ ${ }^{3}$ Institut für Theoretische Physik - Lehrstuhl A, RWTH Aachen, 52056 Aachen, Germany
}

(Dated: October 25, 2018)

\begin{abstract}
We investigate transport through a mononuclear transition-metal complex with strong tunnel coupling to two electrodes. The ground state of this molecule is a singlet while the first excited state is a triplet. We show that a modulation of the tunnel-barrier due to a molecular distortion which couples to the tunneling induces a Kondo-effect, provided the discrete vibrational energy compensates the singlet/triplet gap. We discuss the single-phonon and two-phonon assisted cotunneling and possible experimental realization of the theory.
\end{abstract}

PACS numbers: $85.65 .+\mathrm{h}, 73.23 . \mathrm{Hk}, 73.63 . \mathrm{Kv}, 63.22 .+\mathrm{m}$

The effect of quantized vibrational motion of a singlemolecule device on the electron transport has been investigated intensely. Vibrational effects have been observed in the sequential tunneling regime [1, 2, 3] as well as the strong tunneling regime [4, 5, 6]. Theoretical attention first focused on the weak coupling limit, where exchange of mechanical energy quanta with tunneling electrons (vibration assisted tunneling) 7, 8, 9, 10, 11, 12 and modulation of the tunnel barriers (shuttling) [8, 13. were discussed. In the strong tunneling limit the effect of vibrations on the Kondo-anomaly in the linear conductance was discussed due to assisted tunneling 14, 15, 16 and also due to tunnel-barrier modulation 16]. In this Letter, however, we demonstrate that discrete vibrations through tunnel-barrier modulation can induce a Kondo effect in a transition-metal (TM) organic complex (TMOC) with even number of electrons by compensating for the singlet-triplet splitting at zero bias. Already for molecules of moderate size, many vibrational modes are available, underlining the importance of considering the above effect. Our effect is essentially different from the phonon-assisted Kondo effect in [15, 16]: no strong electron-vibration coupling is required nor special gatevoltage restrictions.

We study the transport through a TMOC with a TM ion secluded in a ligand cage. The cage is in tunnel contact with metallic reservoirs (surface, STM nanotip, or edges of metallic wire in electro-migration or break junction geometry). Fig. 1(a) illustrates this setup. We consider a TMOC with even electron number $N$ fixed by charge and energy quantization. The ground state is supposed to be a spin singlet, and the energy of the lowest triplet excitation $\Delta$ exceeds Kondo temperature $T_{K}$. The linear conductance is thus suppressed. To investigate how intramolecular vibrations may induce transport through a Kondo effect, in the first place one should incorporate a vibronic mode in a generic tunneling Hamiltonian

$$
H=H_{m o l}+H_{\text {res }}+H_{\text {tun }}
$$

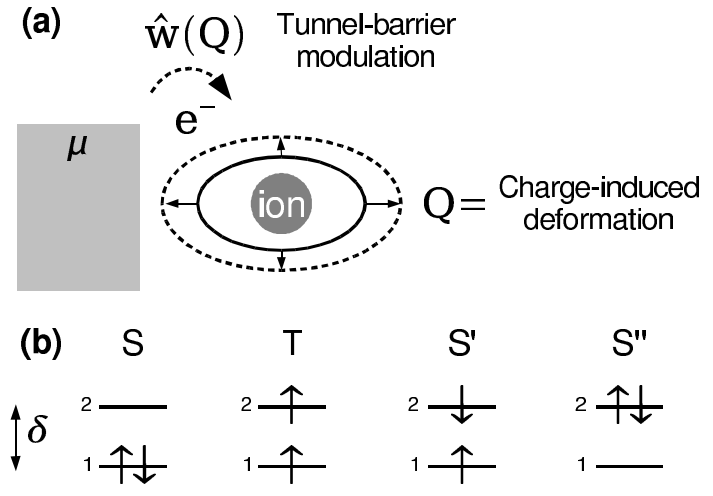

FIG. 1: Schematic situation: (a) Electrode tunnel-coupled to a transition-metal organic complex. Charging of the complex by a tunnel process deforms the outer part of the ligand cage without strongly affecting the direct coordination-sphere of the metal ion and thereby the ligand-field splitting. We assume that the extra electron is localized mainly on the cage. Electrons tunnel onto the ion through the tails of the molecular state centered on the ion, which includes admixtures of the outer shell electronic states. Therefore the main effect of the charging is the modulation of the tunnel barrier between the ion-centered states and electrode. (b) Electronic $e$-type states discussed in the text in order of increasing energy.

Here $H_{m o l}$ includes the $3 d$ electron levels in a ligand field of the cage electrons, the molecular orbitals of these ligands, as well as interactions within the $3 d$ shell and within the cage. One should take into account the three most relevant charge states including their dependence on the vibrational coordinate of the cage $Q$ :

$$
H_{m o l}=H_{Q}^{(N)}+H_{Q}^{(N+1)}+H_{Q}^{(N-1)}+T_{n}
$$

The last term $T_{n}$ is the kinetic energy of the cage distortion. The eigenstates of $H_{Q}^{(N \pm 1)}$ are admixed to those of $H^{(N)}$ by the tunneling $H_{\text {tun }}$ of electrons from the reser- 
voir $H_{r e s}$ :

$$
H_{\text {res }}+H_{\text {tun }}=\sum_{k \sigma} \epsilon_{k} c_{k \sigma}^{\dagger} c_{k \sigma}+\hat{w}_{Q} \sum_{k \mu \sigma}\left(\tilde{d}_{\mu \sigma}^{\dagger} c_{k \sigma}+H . c .\right) .
$$

Effectively a single electrode remains after the standard rotation of electron states [22]. We assume that there is no direct tunneling contact between the $3 \mathrm{~d}$ electrons of the caged TM and the electrodes. However the $3 d-$ orbitals are hybridized with ligand molecular orbitals, and tunneling becomes possible due to small overlap between the "tails" of distorted orbitals $\tilde{d}$ and the lead electrons (see caption to Fig. 1(a) for more details).

We write $H_{m o l}$ for the low-lying many particle states of the TMOC in the compact form

$$
H_{m o l}^{(N)}=\sum_{\Lambda=S, T 0, T \pm} E_{\Lambda}(Q) X^{\Lambda \Lambda}
$$

where $X^{\Lambda \Lambda^{\prime}}=|\Lambda\rangle\left\langle\Lambda^{\prime}\right|$ are so called Hubbard operators describing transitions between the eigenstates $\Lambda, \Lambda^{\prime}$ of the TMOC, and all many-particle effects and their coordinate dependence are included in the energies $E_{\Lambda}(Q)$. Similarly, states of the charged TMOC with $N \rightarrow N \pm 1$ are denoted as $|\gamma\rangle$. The microscopic origin of the lowenergy singlet (S) and triplet ( $\mathrm{T}$ ) is most easily conceived as follows. The evenly occupied ligand cage is in a singlet spin state, and it does not affect the structure of the spin multiplet. We assume that the ligand field of the cage has low symmetry, so that the 5 -fold orbital degeneracy is completely lifted. For instance, for a distorted tetrahedral symmetry of the ligand field we focus on the configuration $d^{2}\left(e^{2}\right)$ with two $e$-states split by $\delta$ due to this distortion. In case of distorted cubic symmetry, the same S/T multiplet arises for the configuration $d^{8}\left(t^{6} e^{2}\right)$. Fig. 团(b) illustrates the states of the TMOC: it is seen that in the case of a weak intrashell exchange $I<\delta$ the ground state is singlet $\mathrm{S}$ and the lowest excitation is the $\mathrm{S} / \mathrm{T}$ transition. The energy difference $\Delta \equiv E_{T}-E_{S}=\delta-I$ is assumed to be larger than the Kondo temperature $T_{K}$. The excitation energies of two singlet excited states $S^{\prime}$ and $S^{\prime \prime}$ are $\delta$ and $2 \delta$, respectively. We ignore below these singlet excitations, since they are not involved in the Kondo tunneling.

We use the simplest approximation of single harmonic vibration mode with frequency $\Omega$. We assume that the relative shifts of the harmonic potentials in different excited and charged states is negligibly small i.e. for all $x=\Lambda, \gamma$ we have $E_{x}(Q) \approx E_{x}+\Omega Q^{2} / 2$ and $T_{n}=\Omega P^{2} / 2$. The coordinate $Q$ is normalized to the zero-point motion. In this weakly non-adiabatic limit vibrations are slow compared to the electron motion on the molecule. No vibrational excitations can be induced by the tunneling in the linear transport regime, unless the distortion of the ligand cage $Q$ affects the tunnel amplitude $\hat{w}(Q)$. Then local phonons can be emitted or absorbed. The tunneling Hamiltonian may be rewritten in the form

$$
H_{t u n}=\hat{w}(Q) \sum_{k} \sum_{\Lambda \gamma \sigma}^{\prime}\left[X^{\Lambda \gamma} c_{k \sigma}+H . c .\right]
$$

(here the second summation is restricted by spin selection rules). This situation is opposite to the anti-adiabatic limit where the vibrations are fast and many phonon excitations are involved in the Kondo exchange [14].
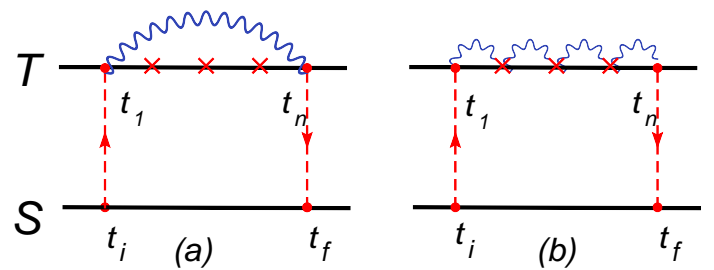

FIG. 2: Two types of phonon-assisted Kondo co-tunneling processes. (a) Virtual phonon absorption initiates a S/T transition, Kondo processes take place in an intermediate triplet state and the phonon is emitted in the end. (b) Every spin flip process in the intermediate triplet state is accompanied by a two-phonon process. Points and crosses denote spin-flip process in the $\mathrm{S} / \mathrm{T}$ and $\mathrm{T} / \mathrm{T}$ channels, $t_{i, f}$ are initial and final times, and $t_{1} \ldots t_{n}$ denote the intermediate co-tunneling acts.

Thus we consider the effect of the modulation of the tunnel amplitude on the linear transport in the Coulomb blockade regime. In this regime one has to eliminate the tunneling term from the Hamiltonian by summing over virtual processes where $N \pm 1$ electrons occupy the molecule and vibrational quanta are excited in these virtual states. This procedure known as a Schrieffer-Wolff (SW) transformation, leads us to a Kondo Hamiltonian (Eq. (6) below) for a S/T multiplet [17] and an oscillator with electron-phonon interaction built into the exchange coupling. According to Ref. 17], transitions within the singlet/triplet spin manifold are described by two vectors $\mathbf{S}$ and $\mathbf{R}$, where $\mathbf{S}$ is the usual spin 1 vector and $\mathbf{R}$ is a vector describing $\mathrm{S} / \mathrm{T}$ transitions. These two vectors are constructed by means of Hubbard operators $X^{\Lambda \Lambda^{\prime}}$ in the following way [17]:

$$
\begin{aligned}
& S^{+}=\sqrt{2}\left(X^{10}+X^{0-1}\right), S^{z}=X^{11}-X^{-1-1}, \\
& R^{+}=\sqrt{2}\left(X^{1 S}-X^{S-1}\right), R^{z}=-\left(X^{0 S}+X^{S 0}\right) .
\end{aligned}
$$

The effective Hamiltonian arising after the SW transformation has the form

$$
H_{\text {eff }}=H_{r e s}+\frac{1}{2} \Delta \mathbf{S}^{2}+\hat{J}_{S} \mathbf{S} \cdot \mathbf{s}+\hat{J}_{R} \mathbf{R} \cdot \mathbf{s}+\frac{\Omega}{2} P^{2}
$$

The electron spin operator is given by the conventional expansion $\mathbf{s}=\frac{1}{2} \sum_{k k^{\prime}} \sum_{\sigma \sigma^{\prime}} c_{k \sigma}^{\dagger} \boldsymbol{\tau}_{\sigma \sigma^{\prime}} c_{k^{\prime} \sigma^{\prime}}$ where $\boldsymbol{\tau}$ is a Pauli vector. The exchange coupling constants $\hat{J}_{S, R}(Q)$ are estimated as $\hat{J}_{S}(Q) \approx \sum_{\gamma}|\hat{w}(Q)|^{2} /\left|E_{T}-E_{\gamma}\right|$, and $\hat{J}_{R}=\alpha \hat{J}_{S}$. Here $\alpha<1$ is a coefficient arising because of 
admixture of singlet states $S^{\prime}, S^{\prime \prime}$ to the ground state [17]. In the weakly non-adiabatic regime the kinetic energy in the denominator has been neglected and the nearly identical $Q$ dependence are practically cancelled out in the addition energies. Thus, no multiphonon replicas appear in the denominators of $\hat{J}_{S, R}$ unlike Ref. 14].

The main source of phonon emission/absorption in our case is the tunneling rate $|\hat{w}(Q)|^{2}$. Expanding it in the quantized displacement operator $Q=\left(b^{\dagger}+b\right) / \sqrt{2}$ we come to phonon assisted exchange vertices presented in Fig. 3] In accordance with Fig. 2] we retain only singlephonon processes for $\hat{J}_{R}(Q)$ and only two-phonon processes for $\hat{J}_{S}(Q)$ :

$$
\hat{J}_{S}(Q)=J_{S}+j_{S} Q^{2}, \quad \hat{J}_{R}(Q)=J_{R}+j_{R} Q .
$$

It is obvious that $j_{S} \ll j_{R}$. To draw these vertices we

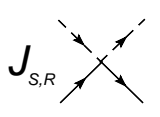

(a)

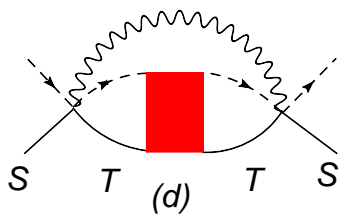

(d)

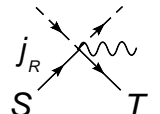

(b)

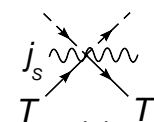

(c)

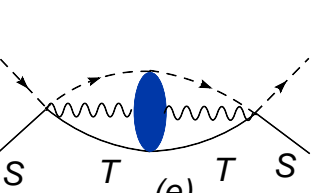

(e)

FIG. 3: (a): Bare exchange vertices $J_{S, R}$; (b) Single phonon correction $j_{R}$ to the vertex $J_{R}$; (c) Two-phonon correction $j_{S}$ to the vertex $J_{S} ;(\mathrm{d}, \mathrm{e})$ Renormalized vertices $\gamma_{1,2}$ corresponding to the processes illustrated by Fig. 2]a), (b), respectively.

used the fermionic representation for the operators (5)

$$
\begin{array}{ll}
S^{+}=\sqrt{2}\left(f_{0}^{\dagger} f_{-1}+f_{1}^{\dagger} f_{0}\right), & S^{z}=f_{1}^{\dagger} f_{1}-f_{-1}^{\dagger} f_{-1}, \\
R^{+}=\sqrt{2}\left(f_{1}^{\dagger} f_{s}-f_{s}^{\dagger} f_{-1}\right), & R^{z}=-\left(f_{0}^{\dagger} f_{s}+f_{s}^{\dagger} f_{0}\right) .
\end{array}
$$

The spin-fermion, electron and phonon propagators are presented in Fig. 3 by solid, dashed and wavy lines, respectively. Physically, Figs 2(a) and 3(d) describe the regime, where the virtual phonon absorbed by the TMOC in the process of co-tunneling brings the energy necessary to compensate the $\mathrm{S} / \mathrm{T}$ gap $\Delta$. Then the Kondo effect develops as multiple spin-flip co-tunneling in the triplet state, and the system returns to the ground state singlet after virtual phonon emission. The vertex corrections are calculated by means of analytical continuation of Matsubara-type diagrams from imaginary axis to the real frequency axis. Summation of all parquet diagrams entering the 4-tail box in Fig. 3(d) gives for $\gamma_{1}$

$$
\gamma_{1} \sim\left(j_{R}\right)^{2} \rho\left[\frac{\log \left(\frac{D}{\max [T,|\Delta-\Omega|]}\right)}{1-J_{S} A \rho \log \left(\frac{D}{\max [T,|\Delta-\Omega|]}\right)}\right]
$$

here $A \sim 1$ is a constant determined by spin algebra. The Kondo temperature extracted from this equation reads

$$
T_{K}^{(1)} \sim D \exp \left(-\frac{1}{A \rho J_{S}}\right)
$$

Here $D$ is the effective width of the electron conduction band and $\rho$ is the density of states on the Fermi level. The second channel illustrated by Figs 2 (b) and 3(e) involves two single-phonon processes compensating $\mathrm{S} / \mathrm{T}$ transitions. The exchange acting in the intermediate triplet states is accompanied by two-phonon processes. Then summation of parquet diagrams entering the 4 -tail vertex in Fig. 3(e) gives for $\gamma_{2}$

$$
\gamma_{2} \sim\left(j_{R}\right)^{2} \rho\left[\frac{\log \left(\frac{D}{\max [T,|\Delta-\Omega|]}\right)}{1-j_{S} A^{\prime} \rho \log \left(\frac{D}{\max [T,|\Delta-\Omega|]}\right)}\right]
$$

The Kondo temperature characterizing this channel depends on the phonon-assisted exchange constant $j_{S}$

$$
T_{K}^{(2)} \sim D \exp \left(-\frac{1}{A^{\prime} \rho j_{S}}\right) \ll T_{K}^{(1)}
$$

One concludes from these calculations that the singlephonon processes are sufficient to compensate the energy of the S/T splitting and induce resonance tunneling through the TMOC provided a local vibration mode with appropriate frequency satisfying the condition

$$
|\Omega-\Delta| \lesssim T_{K}^{(1)}
$$

exists in the cage. One can expect in this case a significant enhancement of the tunnel conductance already at $T>T_{K}^{(1)}$ according to the law $G / G_{0} \sim \ln ^{-2}\left(T / T_{K}^{(1)}\right)[22$, where $G_{0}$ is the conductance at unitarity limit $T \rightarrow 0$.

Thus we formulated in this paper conditions under which phonons are not only involved in Kondo screening but even induce Kondo tunneling. In is worth mentioning that in spite of the fact that the Kondo effect exists in our case only under phonon assistance, the Kondo temperature (12) is the same as in the usual Kondo effect. Since $T_{K}$ is high enough $(\sim 10 \mathrm{~K})$ in electro-migrated junction experiments with a TMOC deposited between contacts [4, 5, 6], the effect predicted in this work seems to be easily observable. The crucial point is the existence of phonon satisfying condition (13) in a TMOC with the $\mathrm{S} / \mathrm{T}$ multiplet as a lowest spin excitation. One should note, however, that even if this condition is not exactly satisfied, one may tune the system by applying the magnetic field. Then the triplet is split, and only the level $E_{T \downarrow}=E_{T}-E_{Z}$ is involved in the phonon induced Kondo tunneling ( $E_{Z}$ is the Zeeman energy). Then $\Delta$ in (13) is substituted by $\Delta_{Z}=\Delta-E_{Z}$, and $E_{Z}$ may be tuned until the inequality is satisfied. Thus the vibration gives rise to a magnetic field induced Kondo effect at Zeeman 
energies which can be much smaller that $\Delta$. The Kondo screening takes place due to the processes presented in Figs 2(a) and 3/d). The only difference is that in this case the effective spin of the TMOC is one half instead of one 20]. The theory of this effect will be presented in a separate publication.

Another way to tune the condition (13) is to stretch the break junction and thereby distort the TMOC and change the frequency $\Omega$. Such mechanical control was demonstrated experimentally for the $\mathrm{H}_{2}$ molecule [25]. There it was also shown that the isotope effect may be used for the same purpose.

The generic feature of the phonon-assisted Kondo screening discussed in this work is that only the virtual phonon excitations enter the co-tunneling amplitude to compensate the $\mathrm{S} / \mathrm{T}$ energy gap $\Delta$, so that the Kondo effect manifests itself as a zero-bias anomaly (ZBA). Another mechanism of such compensation was discussed in Ref. 18], where the energy deficit was covered by the conduction electron acceleration at finite bias. In that case, the Kondo regime arises in non-equilibrium condition, so it is fragile against dephasing effects [18, 19]. In our case the system remains in thermodynamic equilibrium around ZBA, so that the only limiting factor of this sort is the lifetime of local vibration mode, which is usually long enough in comparison with $\hbar / T_{K}$.

To conclude, we demonstrated in this work that phonon emission/absorption can induce Kondo tunneling in a transition-metal organic complex with even electron occupation and a spin singlet ground state, when the conventional Kondo effect is suppressed. Unlike the situation studied in the current literature [14, 15, 16], where the influence of real phonon excitations on the conventional Kondo effect is discussed, and various kinds of side-band satellites due to the polaronic effect are considered, we appeal to virtual phonon excitation, so that the system remains in a quasi-elastic tunneling regime. One of essential ingredients of our theory is that we use the dynamical symmetry of the TMOC, which characterizes both the spin algebra of localized spin itself and transitions between various levels of different spin multiplets [17. In our case the dynamical symmetry group is $S O(4)$.

Since the tunnel contact between magnetic ion and metallic reservoir in TMOC is mediated by a ligand cage, one may be sure that the relevant vibration excitations are the local phonons characterizing this cage. Although we confined ourselves to a specific model with two electrons in an $e$ subshell, the mechanism is quite general. The theory may be easily modified for any system with the same structure of the lowest spin multiplets. One of such examples is the so called "Fulde molecule" 21] schematically representing the spectra of lanthanocenes, where the rare-earth magnetic ion is sandwiched between two rings of $\mathrm{CH}$ radicals. In that case the number of electrons in the cage and in the $4 f$-shell is odd. Another candidate is the endofullerene family with atoms [23] or magnetic ions within a carbon cage 24]. The theory of vibration-induced Kondo effect may be also generalized for the case of degenerate modes and for more complicated spin-multiplets including half-integer spins. These issues will be discussed in forthcoming publications.

This work is partially supported by the SFB-410 and ISF grants. MK acknowledges support through the Heisenberg program of the DFG. Part of this work is done during KK's stay in the Max Planck Institute of Complex Systems, Dresden. MRW acknowledges financial support through the EU RTN Spintronics program HPRN-CT-2002-00302.

[1] H. Park, et al., Nature 407, 52 (2000).

[2] A. N. Pasupathy, et. al., Nano Lett. 5 (2005).

[3] B. J. LeRoy, S. G. Lemay, J. Kong, and C. Dekker, Nature 432, 371 (2004).

[4] J. Park, et al., Nature 417, 722 (2002).

[5] L. H. Yu, et al., Phys. Rev. Lett. 93, 266802 (2004); L. H. Yu and D. Natelson, Nano Lett. 4, 79 (2004).

[6] H. S. J. van der Zant, et. al., Faraday Discuss., available online, (to appear in 2006).

[7] D. Boese and H. Schoeller, Eur. Phys. Lett. 54, 668 (2001).

[8] K. D. McCarthy, N. Prokofev, and M. T. Tuominen, Phys. Rev. B 67, 245415 (2003).

[9] S. Braig and K. Flensberg, Phys. Rev. B 68, 205324 (2003).

[10] A. Mitra, I. Aleiner, and A. J. Millis, Phys. Rev. B 69, 245302 (2004).

[11] J. Koch and F. von Oppen, Phys. Rev. Lett. 94, 206804 (2005).

[12] M. R. Wegewijs and K. C. Nowack, in: Focus on NEMS, New J. Phys. (online), Eds. R. Blick and M. Grifoni.

[13] D. Fedorets, Phys. Rev. B 68, 033106 (2003); D. Fedorets et al., Phys. Rev. Lett. 92, 166801 (2004).

[14] J. Paaske and K. Flensberg, Phys. Rev. Lett. 94, 176801 (2005).

[15] P. S. Cornaglia, H. Ness, and D. R. Grempel, Phys. Rev. Lett. 93, 147201 (2004).

[16] P. S. Cornaglia, D. R. Grempel and H. Ness, Phys. Rev. B 71, 075320 (2005).

[17] K. Kikoin and Y. Avishai, Phys. Rev. Lett. 86, 2090 (2001); Phys. Rev. B 65, 115329 (2002).

[18] M.N. Kiselev, K. Kikoin and L.W. Molenkamp, Phys. Rev. B 68, 155323 (2003).

[19] A. Rosch, J. Paaske, J. Kroha, and P.Wölfle, Phys. Rev. Lett. 90, 076804 (2003).

[20] M. Pustilnik, Y. Avishai, and K. Kikoin, Phys. Rev. Lett. 84, 1756 (2001).

[21] C.-S. Neumann, P. Fulde, Z. Phys. B, 74, 277 (1989); M. Dolg et al, J. Chem. Phys. 94, 3011 (1991).

[22] L. I. Glazman and M. E. Raikh, JETP Lett. 47, 452 (1988).

[23] T. Almeida Murphy et al, Phys. Rev. Lett. 77, 1075 (1996)

[24] R. D. Johnson, et al., Nature 355, 239 (1992).

[25] D. Djukic, et al., Phys. Rev. B 71, 161402(R) (2005). 